\title{
DER TEXT - EIN MAKROSPRECHAKT?
}

\section{Einleitendes:}

Im folgenden Beitrag wird mittels einer kurzen Textanalyse auf die Möglichkeiten und Grenzen einer Übertragung der Sprechakttheorie auf den Text eingegangen. Dabei wird von dem zentralen Begriff der Illokutionshierarchien ausgegangen.

\section{Allgemeines:}

Wenn Sprechen als eine Sonderform der Kommunikation regelgeleitetes Handeln bedeutet (vgl. Beisbart et al. 1976: 137), dann sind nicht nur einzelne isolierte Äußerungen als Sprachhandlungen zu betrachten, sondern es muß das Grundkonzept der Sprechakttheorie auch auf Texte übertragbar sein, denn wenn wir miteinander kommunizieren, dann geschieht dies bekanntlich auf der Textebene. Diese Behauptung hat nicht die Ambition, die Sprechakttheorie von Austin und Searle in irgendeiner Weise zu bestreiten, sondern es geht vielmehr darum, anhand einer kurzen Textanalyse zu demonstrieren zu versuchen, wie die Erkenntnisse der Sprechakttheorie auf den Text anwendbar sind, bzw. auch zu überprüfen zu versuchen, wo einer solchen Parallelziehung Grenzen gesetzt sind. Schon die einfache Feststellung, daß zwischen Sätzen und Sprechakten keine 1:1-Relation herrscht (vgl. Beisbart et al. 1976: 166) berechtigt zu der Annahme, daß Sprechakte als "kleinste Einheit/en/ der Kommunikation" (vgl. Sowinski 1983: 75) als eine übersatzmäßige Kategorie zu betrachten sind und umgekehrt - Texte "in die Nähe zum Sprechakt" (Vater 1992: 16) geraten. Texte sind komplexe Sprechakte, sie bestehen aus Sprechaktfolgen (vgl. Sowinski, a.a.O.). Die Problematik, wie diese Sprechaktfolgen aufeinander abgestimmt sind, ob dabei Gesetzmäßigkeiten und welche eine Rolle spielen und wie sich das auf die Textsortenspezifik auswirken mag, soll in der folgenden Musteranalyse erörtert werden. 


\section{Der Mustertext: Fünf Todsünden}

Gehaltsverhandlungen sind immer diplomatische Gratwanderungen. Was Sie vermeiden sollten.

I. 1. Erpressungsversuche sind für die meisten Chefs ein rotes Tuch. 2. "Wenn Sie mir nicht mehr bezahlen, dann kündige ich!" ist das schlechteste Argument.

II. 3. Gehaltsvergleiche wie "Frau Schmidt verdient mehr als ich ..." bringen nichts. 4. Das dürfen Sie eigentlich nicht wissen. 5. Außerdem spielt es keine Rolle.

III. 6. Betriebsfeiern sind tabu. 7. Sie bitten den Vorgesetzten "ganz spontan" um mehr Geld, weil er dort offener wirkt - und am nächsten Tag erinnert er sich nicht mehr daran.

IV. 8. Mitleid zieht nicht. 9. Wenn Sie Schulden haben oder wenn die Miete ständig steigt, ist das Ihre Privatsache. 10. Setzen Sie damit nicht den Arbeitgeber unter Druck.

V. 11. Spitze Bemerkungen über das mickrige Gehalt lassen Thren Chef kalt, denn er weiß: 12 . Wer viel jammert, der zieht sowieso nie die Konsequenzen.

Es ist dies ein Text im Text, weil er gekoppelt mit einem umfangreicheren Beitrag im Berufsjournal der Zeitschrift "freundin" (Nr. 22/94, 12.10.94) erschienen ist, und zwar in der Sparte "Erfolgs-Strategien". Die Überschrift des umfangreicheren Rahmentextes (der hier aus Platzgründen nicht aufgeführt werden kann), lautet "Mehr Geld? Tips, die sich auszahlen". Dieser umfangreichere Text (im weiteren Text A), der "die optimale Taktik für Verhandlungen" (a.a.O.) um die Gehaltserhöhung bringt, enthält neben einer allgemeinen Einführung vier weitere Unterkapitel: "Die Vorbereitung", "Das Timing", "Das Gespräch" und "Die Ablehnung". Darin werden Strategien entwickelt und unterbreitet ("So gehen Sie am besten vor", a.a.O.), wie man an dieses heikle, an eine Gratwanderung erinnernde Problem einer Gehaltsverhandlung herangehen soll, damit diese komplexe sprachliche Handlung auch gelingt bzw. im Fall einer Ablehnung nicht "der Kopf in den Sand" (a.a.O.) gesteckt werden muß, sondern man sich selbstkritisch und konstruktiv auf neue Verhandlungen vorbereiten kann.

In sprachlicher Hinsicht enthält der Text $\mathrm{A}$ sehr viele Imperativformen und ihre Äquivalente, z. B. Modalverben wie sollen (im Konjunktiv), müssen, können, auch Infinitive, d. h. hauptsächlich Ausdrucksmittel, die unmittelbar den auffordernden intentionalen Charakter, die direktive illokutive Rolle der einzelnen Teilsprachhandlungen signalisieren:

der Imperativ:

überlegen Sie sich vorher, welche Argumente ...; versuchen Sie auch, sich in den Arbeitgeber hineinzudenken; bereiten Sie sich auf Einwände vor; überlegen Sie, wie Sie kontern können; bitten Sie; erwähnen Sie; 
der Infinitiv:

den passenden Termin ausloten; Strategie ausarbeiten; Ziele und Vorstellungen festlegen; den richtigen Gesprächspartner wählen; im Gespräch überzeugen;

die Empfehlung mit "sollte":

dann sollten Sie sich mit Thren Gehaltswünschen lieber ein wenig zurückhalten; Sie sollten nicht mit einer Kollegenschelte reagieren; dabei sollten Sie weder zu bescheiden sein, noch zu hoch pokern; Sie sollten auf jeden Fall mit Ihrem Anliegen zum direkten Vorgesetzten gehen;

das Modalverb "müssen":

dabei müssen Sie Überzeugungsarbeit leisten.

Wie aus dem Obigen zu ersehen ist, geht es hier auf der Äußerungsebene hauptsächlich um direktive Sprechakte, in denen die Intention verfolgt wird, den Hörer $\mathrm{zu}$ einer bestimmten Handlung $\mathrm{zu}$ veranlassen. Bei einer globalen Übersicht muß jedoch eingeräumt werden, daß es sich dabei um einen Text mit beratender Funktion handelt, d. h., der Rezipient wird nicht aufgefordert, die Empfehlungen zu befolgen, sondern es liegt in seinem Ermessen, inwiefern er die erteilten Ratschläge als Information anzunehmen bereit ist.

Hierbei sei auf drei bemerkenswerte Phänomene hingewiesen. Erstens, daß die Akzeptabilität, d. h. die von den Erwartungsnormen abhängigen Einstellungen des Rezipienten, in Beratungstexten eine zentrale Rolle spielt. Zweitens, daß sprachliche Handlungen auf der elementaren Äußerungs- einerseits und auf der Textebene andererseits auseinanderklaffen: die Diskrepanz besteht darin, daß Sprachhandlungen auf der Äußerungsebene hauptsächlich direktiven illokutiven Charakter aufweisen, während die Textebene informativ-assertiv ausgerichtet ist. Drittens: es handelt sich offenbar auf der Textebene um einen indirekten Sprechakt, wobei die Transposition eigenartigerweise in Richtung vom Direktiven (Wort-Welt-Relation) auf der Ausdrucksseite zum Assertorischen (Welt-Wort-Relation) auf der Inhaltsseite verläuft, was für eine Seltenheit gehalten werden kann, denn üblicherweise werden gerade inzitative Intentionen in der kommunikativen Praxis nach Möglichkeit verschiedentlich umschrieben. Direkter Ausdruck als typische Formulierung appellativischer Intentionen ist auf einige Textsorten beschränkt, z. B. auf juridische Texte aus dem Direktivstil (Vorschriften, Verbote usw.), auf Religionstexte (Die Zehn Gebote), auf medizinische Beratungen, auf Gebrauchsanweisungen. Der Rezipient reagiert auf direkt ausgedrückte Aufforderungen im allgemeinen negativ, sie sind nur angemessen, wenn es sich um unumgängliche, streng normierte Verhaltensregeln handelt oder wenn beim Rezipienten ein Interesse an der Befolgung der Vorschriften vorauszusetzen ist, was auch auf den soeben besprochenen Text A zuzutreffen scheint. Auch die Werbesprache ist ein Beispiel dafür, daß direktive Intentionen nur ausnahmsweise direkt versprachlicht werden, im Regelfall dafür jedoch unzählige andere Ausdrucksvarianten ausgeklügelt werden, um den Adressaten als potentiellen 
Käufer ja nicht abzustoßen, sondern nach Möglichkeit zu fesseln und für den Kauff zu gewinnen. (Vgl. Beisbart et al. 1976: 160 f.)

Vor diesem Hintergrund fällt auch unser Mustertext (Text B) auf, der in den umfangreicheren Beratungstext $A$ integriert und in der Zeitschrift optisch davon mit dicken orangefarbenen Strichen hervorgehoben ist. (Vgl. die Bedeutung der Intertextualität als Textualitätskriterium bei de Beaugrande/Dressler 1981: $118 \mathrm{ff.}$.)

Für diesen Text im Text ist zweierlei charakteristisch:

1. Es kommt darin zu einer Situationsverschiebung, zu einer Dichotomie (vgl. Bračič 1993: 57; Vater 1992: 30 verwendet den Terminus "zerdehnte Sprechsituation"): während im Rahmentext A die Kommunikation einerseits hauptsächlich zwischen dem Textautor, der vermutlich ein erfahrener Fachmann, ein Arbeitspsychologe ist, und andererseits zwischen dem zu beratenden Arbeitnehmer verläuft, (mit diesem letzten können wir uns als Leser auch kurzerhand identifizieren), tritt im Text B neben dem Arbeitnehmer auch der Arbeitgeber deutlicher als eine potentielle Figur in der kommunikativen Interaktion auf. Der Texter sieht aus einer Metaebene mögliche Sprachhandlungen zwischen Arbeitnehmer und Arbeitgeber voraus, und zwar so, daß er eine Art Antiregeln formuliert (das erinnert an Tucholskys "Ratschläge für einen schlechten Redner", s. in Tucholsky 1961: 103), mit anderen Worten, gerade jene sprachlichen Handlungen des Arbeitnehmers werden thematisiert, die keinesfalls vollzogen werden dürfen und dadurch metaphorisch als Todsünden charakterisiert sind: sie führen nämlich garantiert zum Mißlingen der Kommunikation, so daß das Scheitern der Sprachhandlung in diesem Fall quasi vorprogrammiert ist.

2. Ratschläge werden auch sprachlich nicht direkt formuliert (einzige Ausnahme ist Satz 10 ("Setzen Sie damit nicht den Arbeitgeber unter Druck"), sondern auf eine indirekte Art: die Sprachhandlungen des Arbeitnehmers werden in kondensierter, nominalisierter Form dem jeweiligen perlokutiven Effekt, d. h. einer jeweils verhängnisvollen Reaktion des Chefs gegenübergestellt. So haben wir einerseits sprachliche Handlungen des Arbeitnehmers wie Erpressungsversuche, Gehaltsvergleiche, das Erwecken von Mitleid, spitze Bemerkungen über das niedrige Gehalt (Betriebsfeiern im 3. Absatz deuten insbesondere auf ein ungeeignetes Situationsmoment in der Kommunikation zwischen Arbeitnehmer und Arbeitgeber hin). Auf der anderen Seite werden perlokutive Teilakte beim Arbeitgeber als abschreckende Beispiele visualisiert: "für die meisten Chefs ein rotes Tuch", "bringen nichts", "der Chef erinnert sich am nächsten Tag nicht mehr daran", "zieht nicht", "lassen Ihren Chef kalt".

In jeder der fünf Todsünden (Absätze I bis V) gibt es ein weiteres Strukturmerkmal: die Explizierung der kondensiert dargestellten Sprachhandlung des Arbeitnehmers durch den Arbeitspsychologen als Textemittenten, begleitet von seinem fachmännischen Argument, weshalb die jeweilige inkriminierte Sprachhandlung des Arbeitnehmers von vornherein zum Scheitern verurteilt ist: 
Im I. Absatz ist "Wenn Sie mir nicht bezahlen, dann kündige ich" eine explizierte Drohung, "ist das schlechteste Argument" dagegen der darauf bezogene kritische Kommentar des Arbeitsberaters.

Im II. Absatz ist "Frau Schmidt verdient mehr als ich ..." eine hypothetisch explizierte vergleichende Sprachhandlung des Arbeitnehmers, worauf wiederum das Argument des Psychologen folgt: "Das dürften Sie eigentlich gar nicht wissen. Außerdem spielt es keine Rolle."

Im III. Absatz ist die Formulierung "Sie bitten den Vorgesetzten 'ganz spontan' um mehr Geld, weil er dort offener wirkt" die Explizierung des Sprechaktes des Bittens und die Formulierung "und am nächsten Tag erinnert er sich nicht mehr daran" der angeschlossene perlokutive Teilakt, der sich mit dem Argument des Textverfassers deckt.

Im IV. Abstaz wird eine hypothetisch explizierte Sprachhandlung, die unter Berufung auf Schulden und die ständig steigende Miete Mitleid erwecken soll, ebenfalls von einem Gegenargument entkräftet: "Wenn Sie Schulden haben oder wenn die Miete ständig steigt, ist das Ihre Privatsache."

Eine Explizierung der nominal kondensierten Sprachhandlung des Arbeitnehmers fehlt im 5. Absatz, dafür ist die Argumentierung des Beratenden um so deutlicher: "denn er (der Arbeitgeber, S.B.) weiß: Wer viel jammert, der zieht sowieso nie die Konsequenzen."

\section{Die Thesen}

Aufgrund der obigen Analyse lassen sich thesenhaft folgende Beobachtungen zusammenfassen:

1. Die theoretischen Prämissen der Sprechakttheorie sind zwar auf Texte anwendbar, jedoch nicht direkt übertragbar.

2. Der Kontakt zwischen Sprecher und Hörer bei elementaren Sprechakten ist auf der Äußerungsebene direkt, während der Text sich verselbständigt, d. h., der Text ist nicht mit dem Sprecher und Hörer "kopräsent" (vgl. Vater 1992: 23). (In der obigen Textanalyse ist dieses Phänomen mit dem Begriff "Situationsverschiebung" bezeichnet worden.)

3. Der Text ist keine willkürliche Sprechaktfolge (vgl. Sowinski 1983: 75), sondern einzelne Sprechaktsequenzen stehen im Text in gegenseitiger Abhängigkeit, so daß hierarchische Beziehungen entstehen (vgl. Sandig 1986: 57, 173), aus denen sich ein Hypersprechakt (Beisbart et al. 1976: 168) oder mit van Dijk ein Makro-Sprechakt (1980: 212) herauskristallisiert, der die wahre (Beisbart et al., a.a.O.) kommunikative Intention durchklingen läßt, ohne daß diese übergeordnete Gesamtillokution mit sprachlichen Mitteln explizit performativ signalisiert werden müßte. 
4. Der Text verfügt ähnlich wie die Einzeläußerung über ein Illokutionspotential (Beisbart et al. 1976: 140, 167), das von der jeweils dominierenden Illokutionsrolle bestimmt wird und zugleich die kommunikative Funktion des Textes festlegt (vgl. Brinker 1988: 18 und Sowinski 1983: 42). Illokutionshierarchien dürften sich somit als nützlich bei der Herausarbeitung von Textsorten-Kriterien erweisen (Beisbart et al. 1976: 167), indem mehrere "token", konkret vorkommende Texte nach Abstraktion von unwesentlichen Merkmalen, in einem "type" fokussiert werden.

5. In unserem Fall geht es um einen Beratungstext mit folgenden konstanten prototypischen Merkmalen:

a) Wissensvermittlung - im hier nicht aufgeführten Textkomplement $A$ ist dies in der Äußerung "So gehen Sie am besten vor" prägnant zum Ausdruck gebracht. Bei solchen Wissensvermittlungen geht es um informierende Teilsprachhandlungen, die zwei weitere Merkmale postulieren:

b) zukünftiger Akt des Adressaten wird erwartet,

c) das Bedürfnis des Adressaten nach Beratung wird subsumiert (z. B. eine Notsituation, in der sich der zu Beratende befinden mag).

Daraus ist ergänzend festzustellen, $d a ß$ in beratenden Texten nicht nur die Akzeptabilität und die Intertextualität als Textualitätskriterien (vgl. de Beaugrande/ Dressler 1981) eine wichtige Rolle spielen, sondern darüber hinaus auch die Informativität und die Situationalität.

Variable Parameter von Beratungstexten ergeben $u$. a. folgende Variationen des Prototyps:

Beratungstexte im Berufsleben (Kommunikationsbereich): medizinische Beratungstexte, psychologische Beratungstexte, Modeberatungen, kulinarische Beratungstexte (Kochrezepte), Gebrauchsanweisungen, touristische Prospekte.

Beratungstexte können schriftlich und mündlich sein, dialogisch (Beratungsgespräch), monologisch (Kanal, Realisationsweise).

Der jeweiligen Kombination von Merkmalen eines prototypischen Beratungstextes liegt das Bestreben des Textproduzenten zugrunde, einen Beratungstext so zu gestalten, daß er beim Rezipienten optimal ankommt. Diese Beschaffenheit des Textes, beim Rezipienten potentiell eine Handlung als Reaktion auszulösen, möchte ich mit dem Begriff Perlokutionspotential des Textes bezeichnen. Dieses sog. Perlokutionspotential des Textes hängt ab von der richtigen Einschätzung der kommunikativen Situation seitens des Textproduzenten (Adressatenbedürfnisse u.a.m.) sowie von dessen Fähigkeit, innertextuelle Merkmale mit den außertextuellen Gegebenheiten in Einklang zu bringen. Es gilt jedoch auch auf der Textebene, daß dieselbe illokutionäre Rolle verschiedene Perlokutionen hervorrufen kann (vgl. Beisbart et al. 1976: 143), so daß zwischen dem Illokutionspotential eines Textes einerseits und dem Perlokutionspotential desselben Textes andererseits keine 1:1- Relation nachweisbar ist. 


\section{Bibliographie:}

De Beaugrande, Rober-Alain/Dressler, Wolfgang Ulrich (1981): Einführung in die Textlinguistik. Tübingen.

Beisbart, Ortwin et al. (1976): Textlinguistik und ihre Didaktik. Donauwörth.

Bračič, Stojan (1993): Kommunikative Funktion der gegenwärtigen deutschen Umgangssprache in Pressereiseerzählungen. Frankfurt am Main, Berlin, Bern, New York, Paris, Wien.

Brinker, Klaus (1988): Linguistische Textanalyse. Eine Einführung in Grundbegriffe und Methoden. Berlin.

van Dijk, Teun A. (1980): Textwissenschaft. Tübingen.

Sandig, Barbara (1986): Stilistik der deutschen Sprache. Berlin, New York.

Sowinski, Bernhard (1983): Textlinguistik. Stuttgart, Berlin, Köln, Mainz.

Tucholsky, Kurt (1961): Zwischen gestern und morgen. Reinbek bei Hamburg.

Vater, Heinz (1992): Einführung in die Textlinguistik. München.

Povzetek

BESEDILO - MAKRO GOVORNO DEJANJE?

Teoretične premise teorije o govornih dejanjih je mogoče uporabiti tudi za besedila, vendar niso kar avtomatično prenosljive na besedilno raven. Medtem ko je stik med govorcema na ravni posameznih izjav neposreden, se besedilo "osamosvoji", se pravi, da časovno in krajevno od govorcev ni odvisno. Besedilo ni naključen splet posameznih govornih dejanj, ampak so le-ta $v$ besedilu praviloma razporejena na podlagi hierarhičnih razmerij, ki zagotavljajo, da besedilo deluje kot celota $\mathrm{z}$ nekim razpoznavnim, hierarhično nadrejenim govornim dejanjem. Njegov ilokucijski potencial se tem bolj ujema z njegovim perlokucijskim potencialom, čim bolj dognan je bil proces besediljenja. 